Arq. Bras. Med. Vet. Zootec., v.67, n.3, p.873-881, 2015

\title{
Reconhecimento de cores de objetos e de alimentos de cromaticidades opostas por pintos de corte
}

[Recognition of colors of objects and food opposite chromaticities by broiler chicks]

\author{
D.P.B. Fernandes ${ }^{1}$, I.J.O. Silva ${ }^{1}$, A.C. Nazareno ${ }^{1}$, A.C. Donofre ${ }^{1}$, K.B. Sevegnani ${ }^{2}$ \\ ${ }^{1}$ Universidade de São Paulo - Piracicaba, SP \\ ${ }^{2}$ Universidade Estadual Paulista "Júlio de Mesquita Filho" - Registro, SP
}

\begin{abstract}
RESUMO
Objetivou-se com esta pesquisa avaliar, por meio de testes de preferência, a capacidade de percepção de pintos de corte no reconhecimento de objetos e alimentos de diferentes cores. A pesquisa foi realizada no Brasil e consistiu no condicionamento de 60 pintos de um dia sexados, sendo 30 pintos machos e 30 pintos fêmeas, durante três dias, com esferas azuis e alimento vermelho, cores escolhidas por terem cromaticidades opostas. Após a fase de condicionamento, foi realizado um teste de preferência, no qual os animais eram colocados individualmente no centro de uma arena de teste com quatro opções, sendo elas: alimento vermelho; alimento azul; esferas vermelhas; esferas azuis. Cada ave foi avaliada durante 10 minutos. Foram registrados: o período de latência para a primeira bicada; o número de bicadas; a quantidade de bicadas sequenciais em cada tipo de objeto ou alimento; o tempo total da ave em cada compartimento ocupado e o primeiro compartimento ocupado. De acordo com os resultados, pintos fêmeas são menos inibidas quando em contato com uma nova situação em relação aos machos. Entretanto, os machos foram capazes de estabelecer um padrão de ambiente na fase de condicionamento e optaram pelo compartimento que estava mais próximo daquela situação associando, da maneira correta, a cor azul à esfera e a cor vermelha ao alimento. No geral, entre os tipos de cores de objetos e entre os tipos de cores de alimentos, as aves demoraram menos tempo para a decisão da escolha do alimento e da esfera azul, evidenciando-se maior atração, em um primeiro momento, por objetos ou alimentos dessa cor. Entre as duas cores de esferas, menor período de latência, maior número de interações, bem como maior tempo de permanência no local estiveram relacionados à esfera azul, verificando-se total preferência por esse objeto. Ao avaliar os tipos de cores de alimentos, foi possível observar que as maiores médias relacionadas ao número de bicadas e de bicadas sequenciais ocorreram com o alimento vermelho. Conclui-se, portanto, que as aves reconheceram o alimento vermelho e o objeto azul, sendo a cor azul a de maior atratividade para pintos de corte em relação à vermelha.
\end{abstract}

Palavras-chave: cognição animal, reconhecimento de objetos, condicionamento, percepção de cores

\begin{abstract}
The aim of this research was to evaluate, by preference tests, the ability of perception of broiler chicks in the recognition of objects and foods of different colors. The survey was conducted in Brazil and consisted in the conditioning of 60 sexed one-day-old chicks using 30 male chicks and 30 female chicks for three days with blue balls and red food colors chosen for having opposed chromaticities. After the conditioning phase, a preference test was performed, in which the animals were placed individually in the center of an arena test with four options: red food, blue food, red balls and blue balls. Each bird was measured for 10 minutes. Latency to the first peck and the number of pecks, the number of sequential pecks at each object type or food, the total time the bird in each occupied compartment and the first occupied slot were recorded. According to the results, female chicks are less inhibited when in contact with a new situation when compared with males. However, males were able to establish a standard environment in the conditioning phase and opted for the compartment which was closer to that situation, associating the blue
\end{abstract}

Recebido em 7 de abril de 2014

Aceito em 27 de fevereiro de 2015

E-mail: danifernandes@usp.br 


\section{Fernandes et al.}

color to the ball and red color to the food. Overall, among the types of object colors and between types of food colors, the birds took less time for the decision on the choice of blue food and blue ball, showing a greater attraction, at first, to this color. Between the two ball colors, shorter latency period, greater number of interactions as well as more time spent on site were related to the blue sphere, verifying greater preference for this object. When evaluating the types of food colors, it was observed that the highest averages related to the number of pecks, and sequential pecks occurred with red food. Therefore, it is concluded that the birds recognized the red and blue food object, with the blue color having greater attractiveness for broiler chicks when compared to red.

Keywords: animal cognition, object recognition, conditioning, color perception

\section{INTRODUÇÃO}

O estudo das habilidades cognitivas de pintos de corte recém-nascidos, utilizando diferentes cores de objetos e de alimentos, permite verificar o nível de percepção do animal em relação ao ambiente em que ele se encontra, bem como compreender a sua capacidade de retenção de memória e aprendizado, o que contribui para o reconhecimento dos objetos que estão a sua volta.

A cognição ajuda o animal a lidar com o mundo exterior de uma forma flexível (Duncan, 2006). Assim, ao se descobrir as habilidades cognitivas dos animais, isto é, a capacidade de aprender e compreender os progressos relacionados ao seu nível de raciocínio lógico, é possível obter informações sobre o intelecto dos mesmos e os direcionar em suas tomadas de decisões e escolhas (Griffin e Speck, 2004; Dawkins, 2006).

Com a avaliação da cognição, é possível determinar o nível de consciência do animal. A consciência seria como uma "visão interior", que permite que o animal tenha conhecimento de si mesmo, como as suas sensações de medo e de dor (Duncan, 2006).

Na determinação da consciência do indivíduo, é verificado se este é capaz de avaliar e deduzir o significado de uma situação em relação a si mesmo durante um curto espaço de tempo. $\mathrm{O}$ indivíduo não só seria sensível aos estímulos, mas também teria a memória de acontecimentos e imagens mentais de eventos que pode ser usada para tomar decisões adequadas, tanto para evitar consequências negativas como para aumentar as positivas (Broom et al., 2009), fazendo escolhas com ações que eles acreditam serem prováveis de levá-los onde preferem ou evitam o de que não gostam ou têm medo (Griffin e Speck, 2004).

O estudo da preferência de cores por pintos é de suma importância, pois pode auxiliar no manejo do animal por meio da escolha da cor ideal de comedouros, de enriquecimento ambiental e de ração, visto que, de acordo com pesquisas já realizadas por outros autores (Taylor et al., 1969; Miklósi et al., 2002; Ham e Osorio, 2007), a preferência dessas aves por determinados tipos de cores influencia diretamente na sua escolha por alimentos e objetos.

Paralelamente, a interação dos pintos de corte com o ambiente em que estão assume níveis diferentes em relação ao tipo de sexo da ave, principalmente quando estes são colocados em locais diferentes ao que estão familiarizados (Vallortigara, 1996). As fêmeas possuem maior percepção do ambiente à sua volta, melhor desempenho em tarefas de aprendizagem da cor, bem como interagem mais rapidamente com o ambiente em que estão. Entretanto, os machos apresentam bom desempenho na tarefa de aprendizagem de posições de objetos; porém, quando um padrão de ambiente é estabelecido pelo mesmo, este demora mais a interagir com o ambiente (Jones, 1977; Andrew, 1972; Vallortigara, 1996).

De acordo com outros estudos já realizados (Taylor et al., 1969; Salzen et al., 1971), além do reconhecimento de objetos e alimentos pelas cores e formas que possuem, fatores relacionados ao comportamento de imprinting podem influenciar na tomada de decisão da ave para a escolha do que mais a atrai, visto que o imprinting está relacionado ao processo de aprendizagem da ave, determinando as suas preferências (Kirkden et al., 2008). 
Partindo-se do pressuposto de que o reconhecimento de objetos e de alimentos é influenciado pela experiência prévia do animal com os mesmos, bem como pelas cores que possuem, o objetivo do presente trabalho foi avaliar, por meio de testes de preferência, a capacidade de percepção de pintos de corte, machos e fêmeas, no reconhecimento de objetos e alimentos de diferentes cores.

\section{MATERIAL E MÉTODOS}

A pesquisa foi realizada no Laboratório de Ambiência, localizado no Núcleo de Pesquisa em Ambiência (NUPEA) da Escola Superior de Agricultura "Luiz de Queiroz" da Universidade de São Paulo (ESALQ/USP), no município de Piracicaba, São Paulo, Brasil.

Foram utilizados 30 pintos fêmeas e 30 pintos machos com um dia de idade, sendo as fêmeas identificadas por anilhas. As aves foram alojadas em um único círculo de proteção com dois metros de diâmetro, sendo a manutenção da zona de termoneutralidade das aves realizada por meio do aquecimento do ambiente utilizando campânulas. A temperatura do ar foi de $32-34^{\circ} \mathrm{C}$, de acordo com as recomendações de Nascimento et al. (2013).

O presente estudo foi realizado em duas etapas, sendo elas: fase de condicionamento e teste de reconhecimento de objetos e de alimentos.

Com a finalidade de se avaliar o reconhecimento de objetos e de alimentos por pintos de corte, duas cores de ração e de esferas foram utilizadas, sendo elas: azul e vermelha. Para tanto, as rações foram coloridas segundo a recomendação estabelecida por Roper e Marples (1997). Dessa forma, a cor vermelha na ração foi obtida por meio da mistura de $0,5 \mathrm{~mL}$ de corante alimentício vermelho com $1,5 \mathrm{~mL}$ de água, e a coloração azul foi obtida por meio da mistura de $1 \mathrm{~mL}$ de corante azul com $1 \mathrm{~mL}$ de água para $11 \mathrm{~g}$ de ração.

O condicionamento teve duração de 3 dias (Rugani et al., 2009) e consistiu no fornecimento de 60 esferas em isopor de cor azul, com diâmetro de 50mm, distribuídas no círculo de proteção aleatoriamente, e ração de cor vermelha, distribuída em comedouros transparentes. As esferas foram escolhidas em função do estudo desenvolvido por Vallortigara et al. (1998), em que as utilizaram como parceiro social durante testes cognitivos com pintos. A utilização de ração como objeto de estudo foi com o intuito de verificar, também durante os testes, qual a prioridade que a ave daria, quanto à sua alimentação ou aproximação a um companheiro social, quando em um ambiente diferente do qual estava familiarizada (Regolin $e t$ al., 2005). A água oferecida às aves não teve alteração de cor e foi colocada em bebedouros infantis pintados de branco na base, para não influenciar no experimento de cores. $\mathrm{O}$ alimento e a água foram ad libitum.

A escolha das cores do alimento e das esferas foi baseada em estudos já realizados por outros autores. Nesses estudos, a cor preferida por pintos esteve relacionada a alimentos que transmitem comprimentos de onda de luz longos (laranja e vermelho) (Roper e Marples, 1997; Miklósi et al., 2002) e objetos que transmitem comprimento de onda curto (azul), ou seja, cores de cromaticidades opostas, perceptíveis aos olhos das aves (Salzen et al., 1971; Davis e Fisher, 1978) e sensíveis aos cones fotorreceptores da retina desses animais (Osorio et al., 1999).

Após a fase de condicionamento, procedeu-se à fase de teste utilizando uma caixa (arena) para testar os animais individualmente. A arena de teste foi construída em madeira branca nas laterais e papel branco no piso, formando quatro ambientes com dimensões de $30 \mathrm{~cm}$ de profundidade, $20 \mathrm{~cm}$ de largura e $20 \mathrm{~cm}$ de altura.

$\mathrm{O}$ layout da arena em formato " $\mathrm{x}$ " foi desenvolvido para que o pinto, no momento em que fosse deixado no centro da mesma, pudesse observar de maneira clara todas as opções de escolha disponíveis (Fig. 1).

Os compartimentos consistiram em quatro tratamentos, sendo eles: ração de cor azul, ração de cor vermelha, esfera de cor azul e esfera de cor vermelha. Esses tratamentos eram sorteados nos compartimentos a cada troca de animal. 

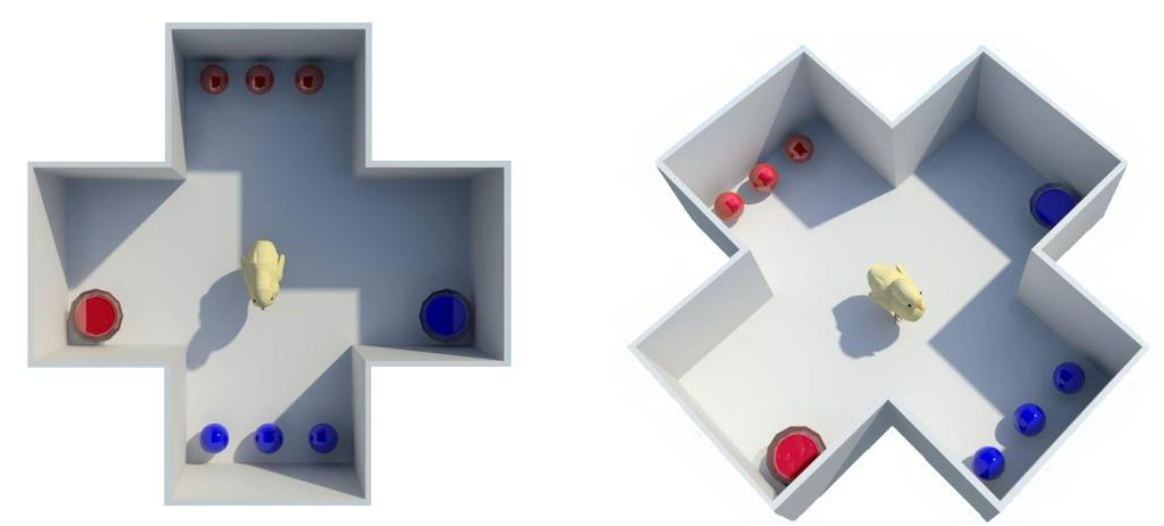

Figura 1. Vista superior e tridimensional da arena de teste utilizada.

Os pintos foram colocados individualmente em um recipiente transparente, no centro da arena, durante 1 minuto, para observarem a área. Após esse procedimento, a ave era liberada para caminhar livremente e escolher o ambiente de preferência.

O período de observação de cada ave foi de 10 minutos. Para avaliação da interação animal/objeto ou animal/alimento, foram registrados o período de latência para a entrada no primeiro compartimento, o número de bicadas, a quantidade de bicadas sequenciais em cada tipo de objeto ou alimento, o tempo total da ave em cada compartimento ocupado e a frequência de troca, uma adaptação da metodologia proposta por Jones et al. (2000).

Adotou-se o delineamento experimental com estrutura de parcela do tipo inteiramente aleatorizada e, como estrutura de tratamento, o esquema fatorial de $2 \times 4$ para avaliação dos comportamentos observados nos diferentes tratamentos (dois sexos e quatro opções de escolha nos compartimentos). Para a análise relacionada ao primeiro compartimento ocupado, foi realizado o teste de Qui-quadrado. Para a análise estatística das variáveis dos demais comportamentos observados, foram utilizados os modelos lineares generalizados, assumindo a distribuição de Poisson, com função de ligação logarítmica (Molenberghs e Verbeke, 2005).

\section{RESULTADOS}

Durante a fase de teste, observou-se que as fêmeas obtiveram um menor período de latência para a escolha do primeiro compartimento a ser ocupado, permaneceram por um período maior dentro dos compartimentos e interagiram mais com o alimento ou objeto de preferência em relação aos machos, cujo fato foi evidenciado pelo maior número de bicadas (Tab. 1). Isso indica que as fêmeas são menos inibidas quando em contato com uma nova situação em relação aos machos.

Tabela 1. Valores médios dos comportamentos observados em relação ao sexo

\begin{tabular}{cccccc}
\hline & \multicolumn{5}{c}{ Comportamentos observados } \\
\cline { 2 - 6 } Sexo & $\begin{array}{c}\text { Latência } \\
\text { (segundo } \\
\text { s) }\end{array}$ & $\begin{array}{c}\text { Número de } \\
\text { bicadas }\end{array}$ & $\begin{array}{c}\text { Número de } \\
\text { bicadas } \\
\text { sequenciais }\end{array}$ & $\begin{array}{c}\text { Tempo total no } \\
\text { compartimento } \\
\text { (segundos) }\end{array}$ & $\begin{array}{c}\text { Frequência } \\
\text { de ocupação }\end{array}$ \\
\hline Fêmeas & $90,52 \mathrm{~b}$ & $0,009 \mathrm{a}$ & $0,003 \mathrm{a}$ & $101,83 \mathrm{a}$ & $2,22 \mathrm{a}$ \\
Machos & $95,78 \mathrm{a}$ & $0,007 \mathrm{~b}$ & $0,003 \mathrm{a}$ & $98,56 \mathrm{~b}$ & $2,17 \mathrm{a}$ \\
\hline Teste do Qui-quadrado & $18,54^{*}$ & $40,31^{*}$ & $0,01 \mathrm{NS}$ & $7,61^{*}$ & $0,07 \mathrm{NS}$ \\
\hline
\end{tabular}

Médias com letras diferentes minúsculas na coluna diferem entre si a nível de 0,05 de probabilidade pelo teste de Qui-quadrado. NS - não significativo, ${ }^{*}$ - significativo a $0,05(\mathrm{P}<0,05)$.

Na Tabela 2, avaliando-se as duas cores de objetos e de alimentos, verifica-se, entre os tipos de cores de objetos e entre os tipos de cores de alimentos, que as aves demoraram menos tempo para a decisão da escolha do alimento e da esfera azul, evidenciando-se maior atração, em um 
primeiro momento, por objetos ou alimentos à sua volta que transmitem comprimento de onda curto.

Entre as duas cores de esferas, menor período de latência, maior número de interações (número de bicadas e bicadas sequenciais), bem como maior tempo de permanência no local estiveram relacionados à esfera azul, verificando-se total preferência por esse objeto, em detrimento dos demais compartimentos.
Ao avaliar os tipos de cores de alimentos, foi possível observar que as maiores médias relacionadas ao número de bicadas e de bicadas sequenciais ocorreram com o alimento vermelho. Dessa forma, embora em um primeiro momento o alimento azul tenha atraído as aves com maior intensidade, a memorização e a familiarização com a ração vermelha ocorridas durante $\mathrm{o}$ período de condicionamento influenciaram na escolha desses animais, fazendo com que os mesmos ficassem mais tempo em contato com esse alimento.

Tabela 2. Valores médios dos comportamentos observados em relação aos compartimentos

\begin{tabular}{cccccc}
\hline & \multicolumn{5}{c}{ Comportamentos observados } \\
\cline { 2 - 6 } Compartimentos & $\begin{array}{c}\text { Latência } \\
\text { (segundo } \\
\text { s) }\end{array}$ & $\begin{array}{c}\text { Número de } \\
\text { bicadas }\end{array}$ & $\begin{array}{c}\text { Número de } \\
\text { Bicadas } \\
\text { sequenciais }\end{array}$ & $\begin{array}{c}\text { Tempo total no } \\
\text { compartimento } \\
\text { (segundos) }\end{array}$ & $\begin{array}{c}\text { Frequência } \\
\text { de ocupação }\end{array}$ \\
\hline Objeto vermelho & $143,54 \mathrm{a}$ & $0,00 \mathrm{~d}$ & $0,00 \mathrm{~d}$ & $46,53 \mathrm{~d}$ & $1,98 \mathrm{bc}$ \\
Objeto azul & $95,78 \mathrm{~b}$ & $0,23 \mathrm{c}$ & $0,02 \mathrm{c}$ & $226,22 \mathrm{a}$ & $2,55 \mathrm{a}$ \\
Alimento vermelho & $79,90 \mathrm{c}$ & $31,98 \mathrm{a}$ & $1,29 \mathrm{a}$ & $128,56 \mathrm{~b}$ & $2,79 \mathrm{a}$ \\
Alimento azul & $67,67 \mathrm{~d}$ & $4,02 \mathrm{~b}$ & $0,20 \mathrm{~b}$ & $74,45 \mathrm{c}$ & $1,66 \mathrm{c}$ \\
\hline Teste do Qui-quadrado $\left(\chi^{2}\right)$ & $1954,65^{*}$ & $4387,86^{*}$ & $170,61^{*}$ & $9227,38^{*}$ & $27,73^{*}$ \\
\hline
\end{tabular}

Médias com letras diferentes minúsculas na coluna diferem entre si a nível de 0,05 de probabilidade pelo teste de Qui-quadrado. NS - não significativo, ${ }^{*}$ - significativo a $0,05(\mathrm{P}<0,05)$.

As menores médias relacionadas ao período de latência estão associadas aos alimentos, indicando que primeiramente os animais procuram o alimento e depois algo que provavelmente os distraia, nesse caso, as esferas.

O maior tempo total no compartimento com as esferas azuis evidencia também que os pintos preferiram o ambiente enriquecido com objetos em vez do alimento, o que pode ser devido à condição de isolamento durante os testes, procurando, com o enriquecimento ambiental proporcionado pelas esferas, aos quais estavam mais adaptados, suprir a falta do companheiro social. Porém, as médias das variáveis respostas relacionadas ao número de bicadas e de bicadas sequenciais foram baixas. Assim como no período de condicionamento, na maioria dos casos, os animais apenas se mantiveram próximos às esferas. Portanto, considera-se, como variável mais apropriada para avaliar a interação com os objetos, o tempo total da ave no compartimento.

Observou-se também que os pintos permaneceram por um maior período de tempo e uma maior frequência de ocupação nos compartimentos com o objeto azul e com o alimento vermelho. Paralelamente, nenhuma interação foi evidenciada com o objeto vermelho, bem como um baixo número de bicadas no alimento azul foi observado. Esse fato comprova que os animais adquiriram um padrão de estímulo particular para si mesmos durante a fase de condicionamento.

Para a variável relacionada ao primeiro compartimento ocupado, não houve interação entre os sexos e os compartimentos $(\mathrm{P}>0,05)$. Entretanto, considerando-se que o primeiro compartimento ocupado seja o mais atrativo para as aves, observou-se que para o sexo feminino não houve diferença estatística entre as escolhas $\left(\chi^{2}=1,73 ; \mathrm{P}>0,05\right)$, ou seja, não há um padrão de preferência entre as fêmeas. Para os machos, a maior atratividade esteve voltada para o compartimento que continha a esfera azul e para o alimento vermelho $\left(\chi^{2}=24,17 ; \mathrm{P}<0,05\right)$. Tal resultado evidencia que esse sexo estabeleceu um padrão de ambiente na fase de condicionamento e optou pelo compartimento que estava mais próximo àquela situação, associando da maneira correta a cor azul à esfera (Fig. 2). 


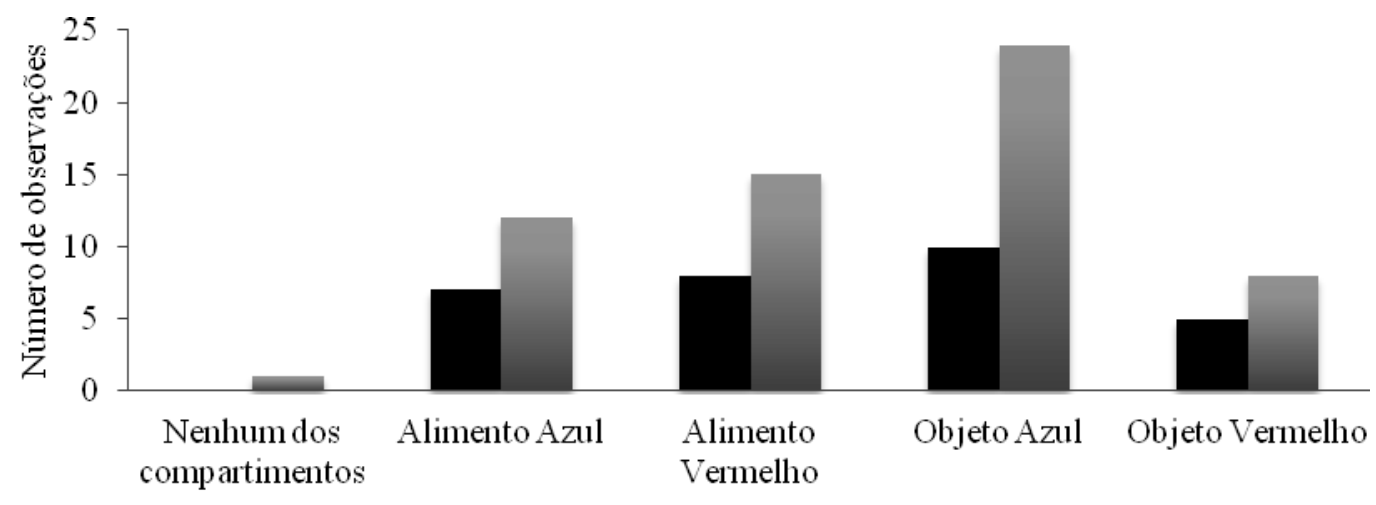

Compartimento de preferência

-Fêmea Macho

Figura 2. Número de observações para o primeiro compartimento ocupado por pintos fêmeas e machos.

Embora se tenha oferecido aos animais opções de escolhas com as quais ainda não haviam tido contato, ou seja, ração azul e esferas vermelhas, estes ainda preferiram a cor da esfera e do alimento utilizado durante a fase de condicionamento, evidenciando total associação da cor vermelha ao alimento, bem como da cor azul à esfera.

\section{DISCUSSÃO}

De acordo com os resultados obtidos neste estudo, verifica-se a preferência de pintos ao objeto de cor azul, devido ao maior tempo de permanência das aves nesse local e menor tempo para abordar o alimento e o objeto da cor mencionada, indicando que a mesma atraiu os animais com maior intensidade em relação às demais opções de escolha.

A aproximação de pintos a objetos e sua relação com a preferência dessas aves foram citadas em outros estudos. Em experimento realizado por Salzen et al. (1971), estes verificaram a preferência de pintos a cores de discos levandose em consideração apenas a aproximação dessas aves aos objetos, se demonstraram algum sinal de contentamento ou mesmo se ficaram quietos quando se aproximavam dos objetos. Ainda, segundo Bolhuis (1999), quando um pinto está perto de um objeto, irá abordá-lo e tentar aconchegar-se a ele. Com base nesses estudos e nos resultados encontrados, o tempo de permanência no local é variável crucial para se entender a preferência por pintos a objetos de diferentes cores.

Os resultados evidenciam também a maior interação da fêmea com o ambiente à sua volta. Entretanto, o primeiro compartimento ocupado pelos machos foi o com esferas azuis e alimento vermelho, com o qual estavam mais familiarizados, ou seja, o mesmo tipo de objeto e de alimento utilizados durante a fase de condicionamento. Esses dados se opõem aos encontrados por Vallortigara (1996), em que os pintos fêmeas, treinados com caixas verdes e vermelhas, apresentaram maior preferência para a cor com que estavam habituadas no momento do teste em relação aos machos. Porém, a partir do $9^{\circ}$ dia, essa maior familiaridade pela cor foi verificada nos machos.

As diferenças de comportamento entre os sexos encontradas no presente trabalho estão de acordo com Jones (1986). Esse autor afirma que pintos fêmeas alimentam-se significativamente mais cedo e apresentam maiores interações com o alimento em relação aos machos. Além disso, apresentam menor inibição comportamental quando colocadas num ambiente novo ou de campo aberto (Jones, 1977). Essas diferenças entre os sexos devem-se à maior quantidade de testosterona nos machos que possui relação com o medo que sentem à nova situação à qual foram expostos, em comparação com as fêmeas (Jones, 1986). 
Neste estudo, observou-se que a tendência dos machos foi visitar primeiramente o ambiente com o qual estavam condicionados, ou seja, adaptados. Esse fato corresponde às observações de Jones (1977) e Andrew (1972). Esses autores afirmaram que, quando um determinado padrão de comportamento foi estabelecido, este possui uma maior duração para os machos em relação às fêmeas, o que influencia no maior tempo de latência para a escolha do primeiro compartimento a ser visitado pelos pintos machos, diante à exposição ao novo ambiente.

A partir dos resultados encontrados, foi verificado que o condicionamento inicial influenciou significativamente na escolha dos animais no período de teste. Comportamento de imprinting semelhante foi relatado em estudo realizado por Salzen et al. (1971). Esses autores verificaram que aves criadas com discos vermelhos os escolheram quando testadas com os estímulos azuis, uma tendência oposta ao grupo controle, sem experiência anterior de estímulos de cor. No mesmo estudo, aves criadas com o estímulo azul mostraram uma forte preferência por azul sobre o vermelho. Também o autor Horn (1993) comenta que, quando os filhotes tornam-se progressivamente mais familiarizados com um objeto, eles tendem a abordar esse objeto com maior intensidade e ao mesmo tempo desenvolver uma tendência a evitar objetos diferentes.

A predisposição à preferência por determinadas cores de alimentos por pintos também é um fator relevante na escolha do alimento. Miklósi et al. (2002), ao testarem rações de coloração azul e laranja, observaram a preferência pelo alimento de coloração laranja, que, assim como o vermelho, transmite luz de comprimento de onda longo. Os autores Roper e Marples (1997) também verificaram que as aves preferem rações de coloração vermelha, em relação às preta e verde.

A falta de interação entre os dois sexos e o compartimento visitado evidenciam que os animais, independentemente do sexo, possuem preferência prévia por determinados tipos de cores de objetos e de alimentos. A maior atração dos animais pelo compartimento onde havia objetos azuis em relação ao alimento vermelho, verificado pela análise do primeiro compartimento visitado pelas aves, está de acordo com as observações efetuadas por Regolin et al. (2005). Segundo o autor, os pintos possuem uma maior habilidade em encontrar o objeto social, em relação ao alimento. Entretanto, essa evidência está associada ao fato de os pintos serem animais que vivem em grupo. Assim, apresentam maior complexidade no funcionamento e desenvolvimento de sua capacidade cognitiva em relação aos animais que não apresentam comportamento social (Broom, 2007). Esses procuram o enriquecimento ambiental para suprirem a falta do companheiro social e como consequência tem-se a minimização dos níveis de estresse decorrentes da separação do grupo (Jones, 1982) e a otimização da alimentação pelo animal (Jones, 1977).

O compartimento contendo esferas vermelhas foi o que as aves demoraram mais a visitar, permaneceram por menor período de tempo e com o qual não foram observadas interações. Embora o período de condicionamento das aves possa ter influenciado em suas escolhas futuras, alguns estudos evidenciam que pode existir a predisposição desses animais à preferência por determinadas cores. Taylor et al. (1969) observaram que pintos preferem ambientes de coloração vermelha, após a exposição dos mesmos a paredes de cores azuis e vermelhas, e utilização de métodos de imprinting convencional. Resultados semelhantes a esses foram verificados pelo autor Jon Cherfas (1978) que, ao aplicar testes de preferência utilizando modelo de labirinto com recompensa para o estímulo azul e sem recompensa para o estímulo vermelho, ou vice-versa, verificou que as aves obtiveram melhor desempenho para a escolha do ambiente com estímulo vermelho (com recompensa). Segundo o autor, essas aves, ao se aproximarem do estímulo azul, têm de primeiro superar sua preferência ao estímulo vermelho, o que leva a resultados de aprendizagem mais lentos.

Em outros estudos, em que foram comparadas as cores vermelha e azul, Ham e Osorio (2007) observaram que pintos preferem objetos com saturação de laranja, e/ou mesmo laranja insaturado, quando comparado com o azul, bem como objetos com saturação de vermelho quando comparados com cores verdes e cinzas. Assim também ocorreu com Herbert e Sluckin (1969), que verificaram preferência a paredes de 
coloração vermelha, quando testadas com as azuis. Porém, em testes com diferentes cores de pisos realizados por Davis e Fisher (1978), não foram encontradas diferenças significativas da preferência entre a cor azul e vermelha.

Observou-se, neste trabalho, clara preferência pela cor azul em detrimento das demais. Entretanto, ao contrário do encontrado, Jones et al. (2000) verificaram maiores períodos de latência de pintos para cordas azuis, quando testadas simultaneamente com cordas de coloração vermelha, azul, verde, amarela e branca, sendo esta última a preferida. Outro fator importante desse estudo foi que, após cinco dias de teste, o período de latência para a escolha da cor preferida (branca) diminuiu e as interações aumentaram. Esse estudo evidencia a capacidade de aprendizagem dessas aves com o passar do tempo e preferência por determinadas cores.

Com base nas variáveis analisadas, observou-se que, entre as cores de objetos, a preferência esteve relacionada à cor azul. Em relação aos alimentos coloridos, considerando-se o número de bicadas, número de bicadas sequenciais e tempo total de permanência em cada compartimento, observou-se que a preferência foi pelo o alimento vermelho. Comparando-se os objetos e os alimentos, foi possível observar que a preferência esteve associada ao objeto azul, devido ao maior tempo de permanência dos pintos no compartimento com esferas azuis.

Evidenciou-se neste estudo que pintos de corte possuem habilidades cognitivas que lhes permitem realizar escolhas que sejam consideradas positivas para os mesmos. No âmbito da senciência animal, estes resultados são importantes para entender como essas aves pensam, e podem servir como base para auxiliar nas definições relacionadas a indicadores de bem-estar de frangos de corte. Paralelamente, trabalhos como este podem auxiliar o produtor na escolha por cores de equipamentos que compõem a instalação na qual pintos de um dia serão criados, bem como nas cores de ração inicial, visto que, neste estudo, a cor mais atrativa foi a azul.

\section{CONCLUSÕES}

Conclui-se que as aves reconheceram o alimento vermelho e o objeto azul, sendo esta última a cor de maior atratividade para pintos de corte em relação à vermelha. Em relação ao sexo, as fêmeas possuem menor inibição comportamental em relação ao ambiente em que se encontram, o que foi evidenciado por maiores valores de interações com os objetos/alimentos.

\section{REFERÊNCIAS}

ANDREW, R.J. Changes in search behaviour in male and female chicks, following different doses of testosterone. Anim. Behav., v.20, p.741$750,1972$.

BOLHUIS, J.J. Early learning and the development of filial preferences in the chick. Behav. Brain Res., v.98, p.245-252, 1999.

BROOM, D.M. Cognitive ability and sentience: which aquatic animals should be protected? Dis.Aquat. Organ., v.75, p.99-108, 2007.

DAVIS, S.J.; FISCHER, G.J. Chick colour approach preferences are altered by cold stress; colour pecking and approach preferences are the same. Anim. Behav., v.26, p.259-264, 1978.

DAWKINS, M.S. Through animal eyes: what behaviour tells us. Appl. Anim. Behav. Sci., v. 100, p. 4-10, 2006.

DUNCAN, I. The changing concept of animal sentience. Appl. Anim. Behav. Sci., v.100, p.1119, 2006.

GRIFFIN, D.R.; SPECK, G.B. New evidence of animal consciousness. Anim. Cognition, n.7, p.5$18,2004$.

HAM, A.D.; OSORIO, D. Colour preferences and colour vision in poultry chicks. Proc. R. Soc. B Biol. Sci., v.274, p.1941-1948, 2007.

HERBERT, M.; SLUCKIN, W. Acquisition of colour preferences by chicks at different temperatures. Anim. Behav., v.17, p.213-216, 1969.

HESS, E.H. Natural preferences of chicks and ducklings for objects of different colors. Psychol. Rep., v.2, p.477-483, 1956. 
HORN, G. Brain mechanisms of memory and predispositions: Interactive studies of cerebral function and behavior. In: HENRY, J.M. (Ed.). Brain development and cognition: a reader. Oxford: Blackwell Publishing, 1993. p.485-513.

JON CHERFAS, J. Simultaneous colour discrimination in chicks is improved by brief exposure to light. Anim. Behav., v.26, p.1-5, 1978.

JONES, R.B. Effects of early environmental enrichment upon open-field behavior and timidity in the domestic chick. Dev. Psychobiol., v.15, p.105-111, 1982.

JONES, R.B. Responses of domestic chicks to novel food as a function of sex, strain and previous experience. Behav. Process, v.12, p.261-271, 1986.

JONES, R.B. Sex and strain differences in the open-field responses of the domestic chick. Appl. Anim. Ethol., v.3, p.255-261, 1977.

JONES, R.B; CARMICHAEL, N.L.; RAYNER, E. Pecking preferences and pre-dispositions in domestic chicks: implications for the development of environmental enrichment devices. Appl. Anim. Behav. Sci., v.69, p.291312, 2000.

KIRKDEN, R.D.; LINDQVIST, C.; JENSEN, P. Effects of domestication on filial motivation and imprinting in chicks: comparison of red junglefowl and White Leghorns. Anim. Behav., v.76, p.287-295, 2008.

MIKLÓSI, Á.; GONDA, Z.; OSORIO, D.; FARZIN, A. The effects of the visual environment on responses to colour by domestic chicks. J. Comp. Physiol. A, v.188, p.135-140, 2002.
MOLENBERGHS, G.; VERBEKE, G. Models for discrete longitudinal data. New York: Springer-Verlag, 2005. 687p.

NASCIMENTO, S.T.; DA SILVA, I.J.O.; MAIA, A.S.C. et al. Mean surface temperature prediction models for broiler chickens - a study of sensible heat flow. Int. J. Biometeorol., p.1-7, 2013.

OSORIO, D.; VOROBYEV, M.; JONES, C.D. Colour vision of domestic chicks. J. Exp. Biol., v.202, p.2951-2959, 1999.

REGOLIN, L.; RUGANI, R.; PAGNI, P.; VALLORTIGARA, G. Delayed search for a social and a non-social goal object by the young domestic chick (Gallus gallus). Anim. Behav., v.70, p.855-864, 2005.

ROPER, T.J.; MARPLES, N.M. Colour preferences of domestic chicks in relation to food and water presentation. Appl. Anim. Behav. Sci., v.54, p.207-213, 1997.

RUGANI, R., FONTANARI, L., SIMONI, E. et al. Arithmetic in newborn chicks. Proc. R. Soc. B Biol. Sci., v.276, p.2451-2460, 2009.

SALZEN, E.A.; LILY, R.E.; MCKEOWN, J.R. Colour preference and imprinting in domestic chicks. Anim. Behav., v.19, p.542-547, 1971.

TAYLOR, A.; SLUCKIN, W.; HEWITT, R. Changing colour preferences of chicks. Anim. Behav. v.17, p.3-8, 1969.

VALLORTIGARA, G. Learning of colour and position cues in domestic chicks: males are better at position, females at colour. Behav. Process, v.36, p.289-296, 1996.

VALLORTIGARA， G.; REGOLIN, L.; RIGONI, M.; ZANFORLIN, M. Delayed search for a concealed imprinted object in the domestic chick. Anim. Cogn., v.1, p.17-24, 1998. 